Case report

\title{
Endovascular treatment of huge saccular abdominal aortic aneurysm in a young Behcet patient: mid-term result Ramazan Kutlu *1, Oner Gulcan ${ }^{2}$, Ahmet Akbulut ${ }^{3}$, Riza Turkoz ${ }^{4}$ and Tamer Baysal 1
}

Address: ${ }^{1}$ Department of Radiology, Inonu university School of Medicine, Turgut Ozal Medical Center, Malatya, Turkey, ${ }^{2}$ Department of Cardiovascular and Thoracic Surgery, Inonu university School of Medicine, Turgut Ozal Medical Center, Malatya, Turkey, ${ }^{3}$ Radiology Clinic, State Hospital, Malatya, Turkey and ${ }^{4}$ Department of Cardiovascular and Thoracic Surgery, Baskent University Adana Hospital, Adana, Turkey

E-mail: Ramazan Kutlu* - rkutlu@inonu.edu.tr; Oner Gulcan - ogulcan@inonu.edu.tv; Ahmet Akbulut - ahmeta@inonu.edu.tru; Riza Turkoz - rturkoz@yahoo.com; Tamer Baysal - tbaysal@inonu.edu.tr

${ }^{*}$ Corresponding author

Published: 22 March 2002

Received: 26 December 2001

BMC Medical Imaging 2002, 2:I

Accepted: 22 March 2002

This article is available from: http://www.biomedcentral.com/I47I-2342/2/I

(C) 2002 Kutlu et al; licensee BioMed Central Ltd. Verbatim copying and redistribution of this article are permitted in any medium for any purpose, provided this notice is preserved along with the article's original URL.

\begin{abstract}
Background: Abdominal aortic aneurysm formation is among the arterial complications of Behcet's disease. Weakness and fragility of aortic walls leads to the development of arterial complications like pseudoaneurysms.

Case Presentation: A case of huge saccular abdominal aortic aneurysm in a young Behcet patient who was successfully treated with endovascular stent graft placement is reported, diagnostic and interventional procedures are discussed, and mid-term follow-up results are presented.

Conclusions: Endovascular treatment of abdominal aortic aneurysm complications of young Behcet patients who are not suitable for open surgery and need intervention could be an alternative treatment modality even without performing preprocedural angiography.
\end{abstract}

\section{Background}

Arterial complications in Behcet's disease are rarely seen and usually involve great arteries like aorta and iliac arteries, and may lead to the perforation of the arterial walls and to the development of the aneurysms and their rupture [1]. Due to weakness and fragility of the aortic wall and arteritis open surgery has some complications like the development pseudoaneurysms. In this case report, an infrarenal huge saccular abdominal aneurysm extending to the left inguinal region in a 32 years old male Behcet patient that was first seen in spinal CT examination performed due to extensive back pain and consequently had a successful stent graft placement is presented.

\section{Case presentation}

A 32 years old male patient with intense back pain admitted to the emergency room. From his history it was learnt that he had Behcet disease and being followed by another institution. Direct roentgenograms revealed a smooth contoured density increase on left side of vertebral column (Figure 1). Lumbar vertebral CT scan revealed significant destruction on the anteriolateral parts of vertebral corpuses between L2 and L4, more prominent on L3 and hypodense area noted. Then dynamic abdominal CT was performed which showed an infrarenal, $10 \times 10 \times 10 \mathrm{~cm}$ saccular aneurysm originating form a $3.5 \times 1 \mathrm{~cm}$ defect on the left posterolateral aspect of abdominal aorta, with thrombus inside (Figure 2A, 2B). Due to the risk of pseu- 


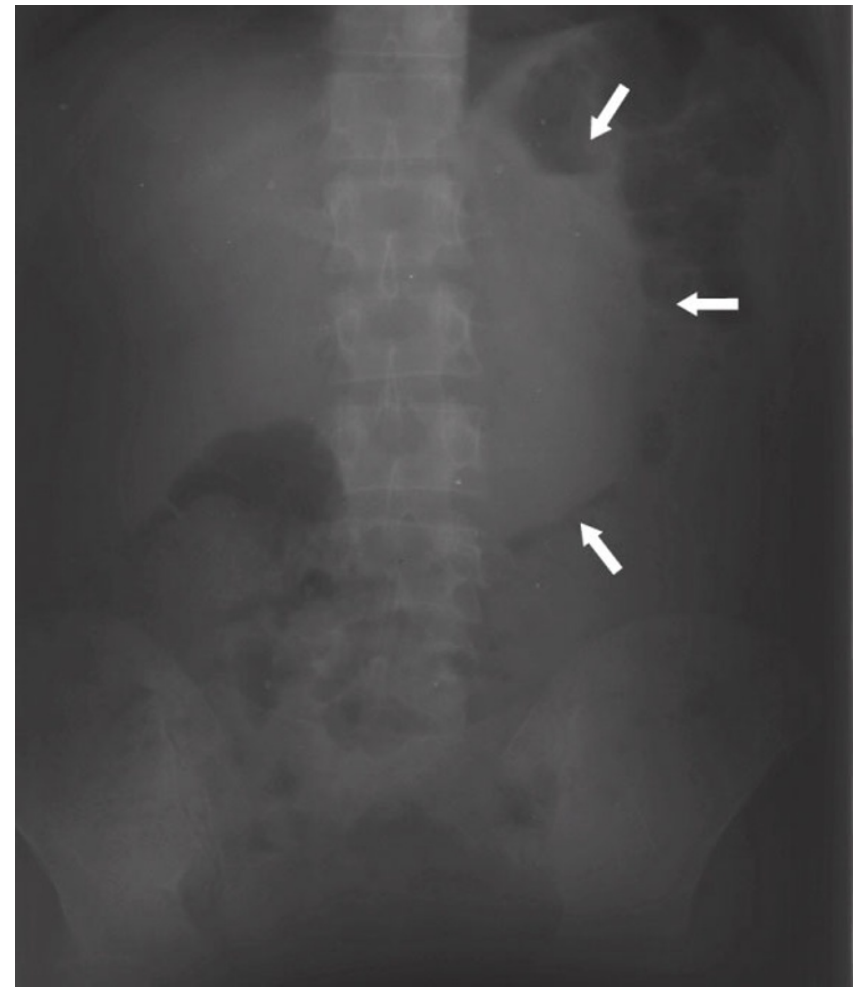

Figure I

Direct roentgenogram of the abdomen shows smooth contoured density increase on left side of vertebral column.

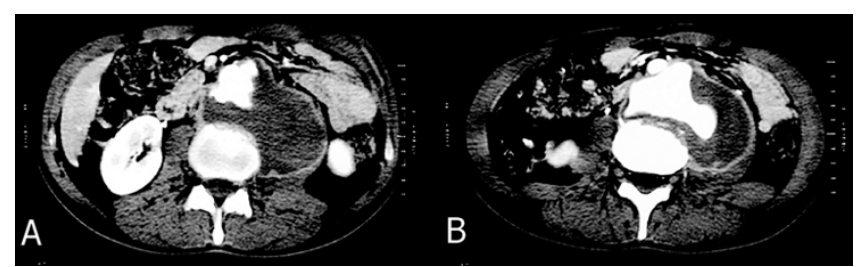

Figure 2

A, B. Abdominal helical CT images show prominent destruction in the left anterolateral portion of the lumbar vertebra corpus and $10 \times 10 \times 15 \mathrm{~cm}$ saccular aneurysm originating from a $3.5 \times 1 \mathrm{~cm}$ defect in the left posterolateral aspect of the abdominal aorta and thrombus inside. The vertebral destruction was probably due to pulsation of the aneurysm.

doaneurysm development digital subtraction angiography (DSA) was not performed and CT angiography (CTA) and $3 \mathrm{D}$ reconstructions were made (Figure 3A, 3B, 3C, 3D, 3E). CT examinations were performed with GE ProSpeed Helical scanner and for the reformat images and 3D reconstructions Advantage Windows version 2.0 Voxtool software on a separate workstation was used. The patient was not considered an ideal candidate for open surgery and endovascular stent graft placement was

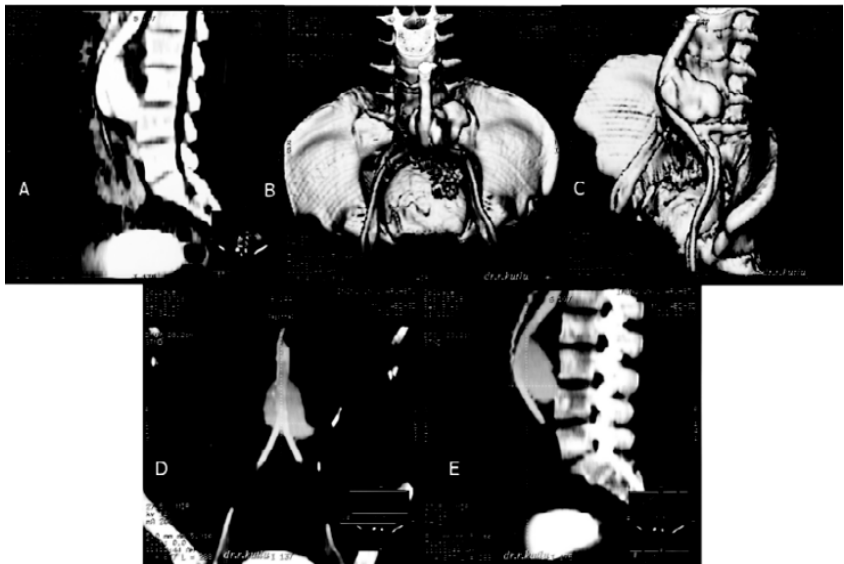

Figure 3

A, B, C, D, E. Sagittal reformat CT image (A), surface shaded display (SSD) 3D images $(B, C)$ and maximum intensity projection (MIP) multiplanar volume reformat (MPVR) images $(D, E)$ show neck of the aneurysm, anterior displacement of the aorta and patency of the iliac bifurcation and iliac arteries.

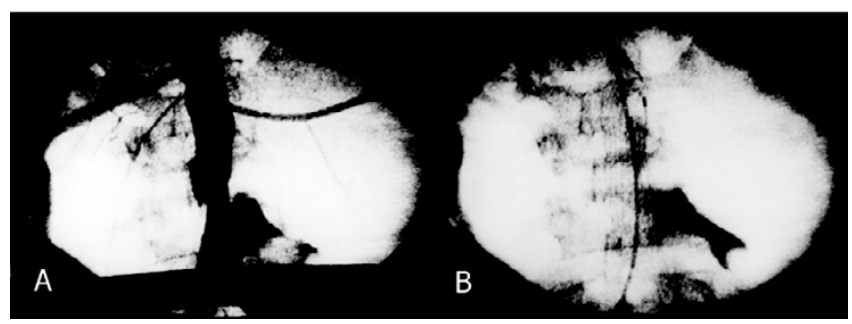

Figure 4

A, B. C-arm fluoroscopy image obtained during the procedure in the operating room show the patency of renal arteries (A) and deployed stent graft (B). Arrowhead points to the contrast material entrapped inside the aneurysm sac.

planned. All the measurements made by using abdominal CT and CTA images that revealed favorable lengths and diameters for endovascular treatment. A nitinol, polyster covered $14 \times 70 \mathrm{~mm}$ stent graft (Stenford Grouppe Valendons fa Nanterre, France), beginning from $1.5 \mathrm{~cm}$ proximal and $1.5 \mathrm{~cm}$ distal to the aortic defect, ordered. Intervention took place in the operating room under spinal epidural anesthesia with the guidance of Schimadzu C-Arm fluoroscopy equipment that had no DSA function. Systemic iv 5000 U heparin was administered. Right common femoral artery was exposed and an arteriotomy was made. A 12 F (75 cm length) introducer was advanced and stent graft was deployed $1.5 \mathrm{~cm}$ below the renal arteries. During deployment systemic arterial pressure dropped to $50 \mathrm{mmHg}$. Intraprocedural control conventional angiography showed the patency of renal arteries and proper position of the deployed stent graft and entrapped contrast 


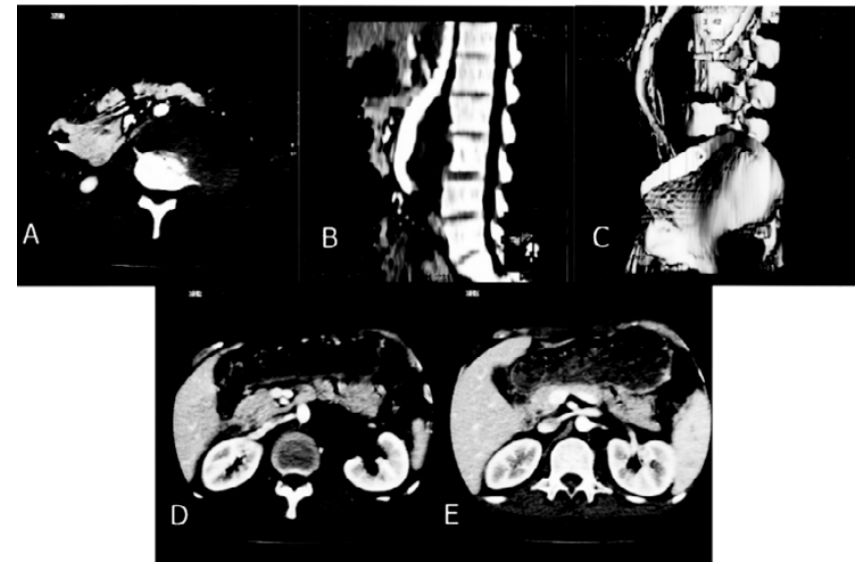

Figure 5

A, B, C, D, E. Abdominal helical CT (A), sagittal reformat CT $(B)$ and SSD images (C) show non-filling of the aneurysm sac and complete sealing of the defect. Abdominal CT images shows right (D) and left (E) patent renal arteries.

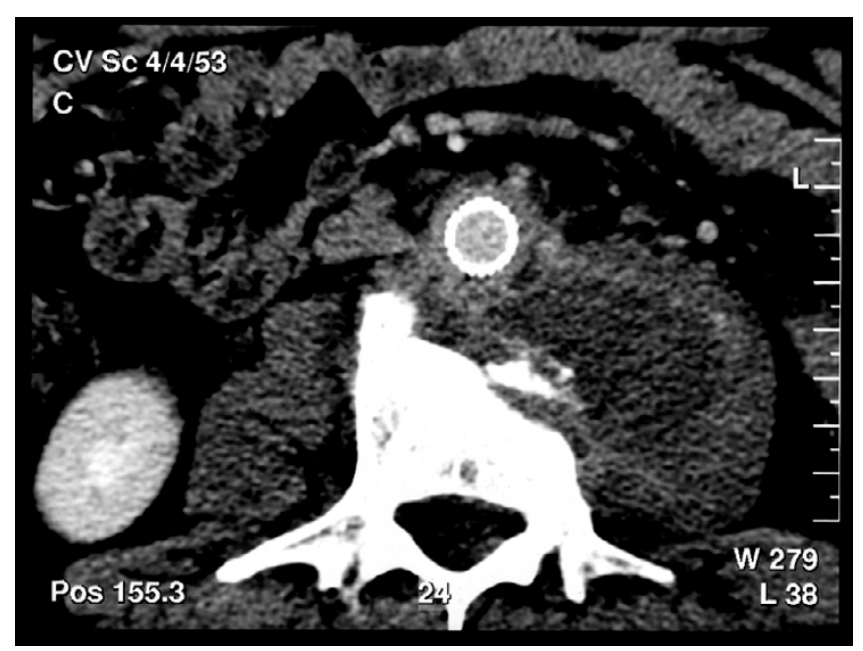

Figure 6

A, B. Control abdominal CT image after 18 months shows a decrease in the size of the aneurysm sac and patency of the stent graft. No endoleak was noted.

material inside the aneurysm sac (Figure 4A, 4B). Arteriotomy site was closed by primary sutures to prevent pseudoaneurysm development and wrapped by woven collagen covered Dacron graft (Hemashield, Meadox Medicals, Oakland, NJ). There was no intervention related intra or post procedural complications. Control abdominal CT examination and 3D reconstructions revealed complete sealing of the aneurysm sac by the stent graft, patency of the renal arteries and visceral arteries and no endoleak was noted (Figure 5A, 5B, 5C, 5D, 5E). No ischemic complications in the lower extremities were encountered. Patient is discharged with steroid therapy and is being followed up serial abdominal CT. After 18 months there were no complications (Figure 6A, 6B).

\section{Discussion}

Behcet disease is first described by Hulusi Behcet, a Turkish dermatologist, in 1937 [2]. It is a multisystemic inflammatory disease that classically causing oral and genital ulcers, and ocular inflammation. It can also affect vascular system. Since it can affect both arteries and veins of all sizes, Behcet vasculopathy is different from other vasculitides [3].

There is no pathognomonic laboratory test or histologic finding specific to Behcet disease. Thus the diagnosis is based on clinical criteria. Various criteria were proposed (like Mason and Barnes, The Behcet's disease Research Committee of Japan, O'Duffy and Goldstein, International Study Group (ISG) etc). The most commonly used criteria of ISG [4] require recurrent oral ulceration plus at least two of the following: recurrent genital ulcerations, eye lesions (like uveitis etc), skin lesions (erythema nodosum, folliculitis etc), positive pathergy test. ISG considered subcutaneous thrombophlebitis, deep vein thrombosis and arterial aneurysms as a criterion. Although these have high specifity for Behcet disease, they are not accepted as criteria due to their low sensitivity.

The etiology of Behcet disease is still not certain but systemic vasculitis has an important role. Perivascular lymphocytic and plasma cell infiltration, endothelial cell proliferation and swelling, disruption of elastic lamina, degeneration of the tunica media and vasculitis of the small vessels in vasa vasorum are among the histologic features of venous and arterial lesions $[5,6]$

Behcet vasculopathy includes arterial and venous thrombosis formations, subcutaneous thrombophlebitis, venous collateralizations, pulmonary artery aneurysm, arterial aneurysm and occlusions [6] Arterial aneurysms are more common than arterial thrombosis. Although aorta is the most common site of involvement pulmonary, femoral, subclavian, popliteal and carotid arteries also could be affected. Histologic examinations of the arterial lesions show vasculitis in the vasa vasorum together with thickening in the media and separation of the elastic fibers, which in turn causes development of aneurysms. [6-8].

Endovascular grafts provide an important alternative to high-risk patients with more invasive surgical procedures. Patients who had endovascular stent graft placement had shorter operating room and hospital stay and less blood loss than open surgery. From the economical stand point the cost of stent graft placement is much higher than open surgery in our country. 
Although numerous improvements in catheter, stent and graft technology have been made, there are still some limitations like the size of delivery systems, endoleaks, position of the renal arteries etc. The success rate of endovascular stent grafting is high $(90 \%$ in low risk patients, $80 \%$ in moderate to high risk patients). Short-term mortality (within the 30 days following the procedure) is reported to be $2.5 \%$ in low risk group, $8 \%$ in high-risk group. Although there is disagreement in reported mortality rates for open surgery [9], there are reports of mortality rates of between $1.6 \%[10]$ to $7,6 \%[11,12]$. As it is common for all new or minimally invasive techniques, there is a learning curve for endovascular stent grafting that affects both the results and costs. Thus for the best results efficiency and experience are needed.

One of the crucial points of endovascular treatment of aneurysms is accurate and precise measurement of aneurysm. Although CT is highly effective, most of the vascular radiologists still would like to have preprocedural angiography. But in Behcet disease vessel wall vulnerability is extremely high and even minor lesions resulting from diagnostic procedures (e.g. intraarterial angiography etc.) could lead to the formation of aneurysms at the puncture site [13]. Due to the fragility of the aneurysm inherent in Behcet patients and the size of aneurysm, we did not perform diagnostic angiography. All measurements based on CT images and 3D reconstructions helped us to better understand and characterize the neck, dimensions and relations of the aneurysm to other arteries.

Endovascular radiological intervention in the treatment of abdominal aortic aneurysm complications of young Behcet patients, in whom surgery is not suitable and intervention is necessary, could be an alternative treatment modality even without performing preprocedural conventional angiography or DSA using the advantages of helical $\mathrm{CT}$ and $3 \mathrm{D}$ reconstruction techniques.

\section{Competing interests}

none declared

\section{Authors' contributions}

Author 1, RK, conceived of the study, carried out the imaging work-up and the endovascular procedure, and drafted the manuscript.

Author 2, OG, carried out the surgical part and participated in drafting the manuscript

Author 3, AA, participated in the imaging work-up and en do vascular procedure.

Author 4, RT, participated in the surgical part, and coordination.
Author 5, TB, participated in the imaging work-up and coordination.

All authors read and approved the final manuscript.

\section{Acknowledgements}

Written consent was obtained from the patient for publication of the patient's details.. The manuscript has been presented in the TURK RAD, 17th National Radiology Congress (27-3I September 2000, Istanbul, Turkey) as a poster presentation.

\section{References}

I. Vasseur MA, Haulon S, Beregi JP, Le Tourneau T, Prat A, Warembourgh $\mathrm{H}$ : Endovascular treatment of abdominal aneurysmal aordds in Behcet's disease. J Vasc Surg 1998, 27:974-976

2. Beçhet $\mathrm{H}$ : Uber rezidiverende aphtose, durch ein Virus verursachte Geschwure am Mund, am Auge und den Genitalien. Derm Wochenschr 1937, 36: I I52-II 57

3. Ghate JV, Jorizzo JL: Behcet disease and complex aphthosis. J Am Acad Dermatol 1999, 40: I- 18

4. International Study Group for Behçet's Disease: Criteria for diagnosis of Behçet's disease. Lancet 1990, 335:1078-1080

5. Bacon PA, Carruthers DM: Vasculitis associated with connective tissue disorders. Rheum Dis Clin North Am 1995, 2 I: I 077-1096

6. Koc Y, Gullu I, Akpek G, et al: Vascular involvement in Behçet's disease. J Rheumatol 1992, 19:402-4I0

7. Matsumoto $T$, Uekusa T, Fukuda $Y$ : Vasculo-Behçet's disease: a pathologic study of eight cases. Hum Pathol 1991, 22:45-57

8. Lie JT: Editorial: Vascular involvement in Behçet's disease: arterial and venous and vessels of all sizes. Rheumatol 1992, 19:34I-342

9. Blankensteijn JD: Mortality and morbidity rates after conventional abdominal aortic aneurysm repair. Semin Interv Cardiol 2000, $5: 7-13$

10. Cruz CP, Drouilhet JC, Southern FN, Eidt JF, Barnes RW, Moursi MM: Abdominal aortic aneurysm repair. Vasc Surg 200 I, 35:335-344

II. May J, White GH, Yu W, et al: Endoluminal repair of abdominal aortic aneurysms: strengths and weaknesses of various prostheses observed in a 4.5-year experience. J Endovasc Surg 1997, 4:|47-|5|

12. Blum U, Voshage G, Lammer J, et al: Endoluminal stent-grafts for infrarenal abdominal aortic aneurysms. N Engl ] Med I997, 336: $13-20$

13. Erpenbach S, Arlart IP, Tremmel-Lehnert C, Hupp T: Abdominal aortic aneurysm in Behçet's disease: treatment with endovascular stent graft. Eur Radiol 200 I, I I:2539-2542

\section{Pre-publication history}

The pre-publication history for this paper can be accessed here:

http://www.biomedcentral.com/1471-2342/2/1/prepub 